\title{
Effect of TrkB-PLC/IP3 pathway on intestinal inflammatory factors and enterocyte apoptosis in mice with colitis
}

\section{Guangmeng $\mathbf{X u}$}

The Second Hospital of Jilin University

\section{Yajuan Sun}

China-Japan Union Hospital of Jilin Univeristy

\section{Huaiqiang $\mathrm{He}$}

The First Hospital of Jilin University

\section{Qiuli Xue}

The First Hospital of Jilin University

\section{Yajie Liu}

The First Hospital of Jilin University

\section{Lihua Dong ( $\nabla$ donglihuac5v@163.com )}

The First Hospital of Jilin Univeristy https://orcid.org/0000-0002-9948-2008

\section{Research article}

Keywords: Colitis, TrkB-PLC/IP3 pathway, Intestinal inflammation, Enterocyte, apoptosis

Posted Date: March 18th, 2020

DOl: https://doi.org/10.21203/rs.3.rs-17639/v1

License: (1) This work is licensed under a Creative Commons Attribution 4.0 International License. Read Full License

Version of Record: A version of this preprint was published at Acta Biochimica et Biophysica Sinica on May 22nd, 2020. See the published version at https://doi.org/10.1093/abbs/gmaa044. 


\section{Abstract}

Background This study aimed to explore the effect of TrkB-PLC/IP3 pathway on intestinal inflammatory factors and enterocyte apoptosis in mice with colitis.

Methods Forty 8-week SPF C57BL/6J mice were randomly and averagely divided into normal group (healthy mice), control group (sham-operated mice), model group (model mice without any treatment), and K252a group (model mice with the treatment of $100 \mu \mathrm{moL} / \mathrm{kg}$ TrkB-PLC/IP3 pathway inhibitor for $5 \mathrm{~d}$ before clysis). Mice in model and K252a groups were used to establish ulcerative colitis models after medication.

Results There were no significant changes of the content of serum tumor necrosis factor- $a$ (TNF- $a$ ) and TNF- $y$ and protein expressions of TNF- $\alpha$ and TNF- $y$ in the colon tissues (all P>0.05), a significant increase of disease activity index, colon mucosa damage index, tissue damage index scores, content and protein expressions of serum interleukin-4 (IL-4) and IL-8, and a significant decrease of content and protein expressions of serum IL-10 (all P $<0.05$ ) in model and K252a groups, as compared to normal and control groups. Mice in model and K252a groups had blocked enterocyte cycle progression, raised apoptosis ratio, significantly increased mRNA and protein expressions of Caspase3, Bax, FasL and Fas, and significantly reduced mRNA and protein expressions of p-TrkB, PLC- $\gamma 1$, IP3 and Bcl-2 (all P <0.05). Moreover, intestinal inflammation and apoptosis induced by colitis in K252a group became more aggravated through the inhibition of TrkB-PLC/IP3 pathway activity.

Conclusions Inhibition of TrkB-PLC/IP3 pathway can promote the expression of intestinal inflammatory factors and enterocyte apoptosis in mice with colitis.

\section{Background}

Ulcerative colitis is a kind of chronic colitis with repeated attack and unknown etiology [1, 2]. Recently, the incidence rate of ulcerative colitis in human beings has increased year by year in China, and ulcerative colitis is considered to be a precancerous symptom of colon cancer. Therefore, it is listed as one of the modern refractory diseases by the World Health Organization $[3,4]$. So far, the exploration of ulcerative colitis is mainly focused on clinical treatment, and there are few studies on related signaling pathway that affecting ulcerative colitis [5-8].

The lesion site of ulcerative colitis is mainly found in the area from the colon submucosa to the entire mucosa, and the main symptoms include ulcer and inflammation. Enterocyte apoptosis may be one cause of ulcer $[9,10]$. The initial symptom of ulcerative colitis is intestinal flora alteration which triggers an abnormal immune response in the body, resulting in high expressions of inflammatory factors in the intestinal mucosa and further generating inflammation [11-13].

In recent years, the effect of TrkB-PLC/IP3 pathway on the intestinal tract has drawn people's attention. TrkB-PLC/IP3 pathway is mainly consisted of protein-tyrosine kinase receptor B (TrkB), phospholipase C 
(PLC) and inositol triphosphate (IP3). After the specific binding of brain-derived neurotrophic factor (BDNF) to TrkB, TrkB is phosphorylated to $\mathrm{p}$-TrkB, which activates the signal transduction of PLC/IP3 in the downstream, thereby playing roles in the increase of intracellular $\mathrm{Ca}^{2+}$ concentration, the enhancement of nerve-muscle excitation conduction, the promotion of cell mobility in the intestinal smooth muscle, and the promotion of intestinal motility [14-19]. At present, there is no research showing the regulatory mechanism of TrkB-PLC/IP3 pathway on colitis. We speculate that TrkB-PLC/IP3 pathway contributes to the regulation of inflammatory response and enterocyte apoptosis for colitis. This study measured the expressions of inflammatory factors and enterocyte apoptosis by establishing ulcerative colitis mice models and treating model mice with TrkB-PLC/IP3 pathway inhibitor K252a, aiming at exploring the effect of TrkB-PLC/IP3 pathway on intestinal inflammatory factors and enterocyte apoptosis in mice with colitis.

\section{Methods}

\section{Laboratory animal}

A total of 40 8-week SPF C57BL/6J mice weighing $22.41 \pm 2.34 \mathrm{~g}$ were purchased from the Beijing HFK Bioscience Co., Ltd., China. The weight and quality of laboratory mice met the national standards, and all mice were fed conventionally. The protocol and procedures employed were ethically reviewed and approved by the Animal Ethics Committee of The Second Hospital of Jilin University (No.D20190013), and experiments were performed in accordance with relevant institutional and national guidelines for the care and use of laboratory animals.

\section{Establishment of colitis mice models}

Twenty mice were randomly selected to establish colitis models, 10 mice were used as sham-operated mice, and 10 mice were fed conventionally. After the mice for establishing colitis models were allowed to acclimate for 1 week, they were induced using oxazolone. Oxazolone was dissolved in $50 \%$ ethanol to prepare $1 \%$ and $3 \%$ oxazolone solution. Mice were anaesthetized with intraperitoneal injection of 100 $\mathrm{mg} / \mathrm{kg} 1 \%$ pentobarbital sodium. The abdomen of sham-operated mice and model mice was shaved, and the skin surface was smeared with $2 \mathrm{~mL} 3 \%$ oxazolone once a day for $5 \mathrm{~d}$ to generate sensitization. Then, $150 \mu \mathrm{L} 1 \%$ oxazolone was infused by inserting a $2 \mathrm{~mm}$ silicone tube approximately $4 \mathrm{~cm}$ from the anus to conduct clysis. The skin surface of sham-operated mice was smeared with $2 \mathrm{~mL} \mathrm{50 \%} \mathrm{ethanol,} \mathrm{and} 150$ $\mu \mathrm{L} 50 \%$ ethanol was used to conduct clysis; the remaining treatment was the same as that in model mice. The body weight loss and diarrhea occurred $24 \mathrm{~h}$ after clysis, indicating a successful modeling. Mice after clysis and healthy mice were bred conventionally under the same rearing condition.

\section{Grouping}

The mice were randomly and averagely divided into four groups: Normal group (healthy mice), control group (sham-operated mice), model group (model mice without any treatment), and K252a group (model mice with the treatment of $100 \mu \mathrm{moL} / \mathrm{kg}$ TrkB-PLC/IP3 pathway inhibitor). Mice in K252a group before 
clysis were intraperitoneally injected with $100 \mu \mathrm{moL} / \mathrm{kg} \mathrm{K} 252 \mathrm{a}$ once a day for $5 \mathrm{~d}$. At $24 \mathrm{~h}$ after modeling, the disease activity index (DAl) of the mice was observed. The mice were anesthetized with administer intraperitoneal injection of $100 \mathrm{mg} / \mathrm{kg}, 1 \%$ sodium pentobarbital. Under anesthesia, eyeballs were picked out to collect peripheral blood. After mice died of excessive blood loss, the colon tissue was taken. The colonic mucosal injury index, histological injury index, and related molecular biological indicators were observed. The Ethics Committee of The Second Hospital of Jilin University had approved this method of euthanasia.

\section{Disease activity index scoring}

Detailed scoring rules on disease activity index (DAl) [8]: After $24 \mathrm{~h}$ of successful modeling the mice were weighed; fecal status was recorded; occult blood was detected; DAl was calculated. DAI was scored by overall consideration of mice weight loss, fecal viscosity and hemafecia status. Scoring was as follows: no weight loss of 0 , weight loss less than $5 \%$ of zero, weight loss of $5 \%-10 \%$ of two points, weight loss of $10 \%-15 \%$ of three points, weight loss greater than $15 \%$ of four points; normal fecal viscosity of zero, loose stools of two points, diarrhea of four points; occult hemafecia of two points, revealed hemafecia of four points. The total score of weight loss, fecal viscosity and hemafecia was divided by three to obtain DAI score.

\section{Colon mucosa damage index scoring}

Detailed scoring rules on colon mucosa damage index (CMDI) [8]: After mice were sacrificed under anesthesia, the morphological changes of colon mucosa were observed with the naked eye to score CMDI. No mucosa damage was scored as zero; mild edema and hemorrhage on the surface of intestinal mucosa were scored as one point; moderate hyperemia, edema and erosion in the intestinal mucosa were scored as two points; severe hyperemia and edema, tissue necrosis, ulcer with a diameter of less than 1 $\mathrm{cm}$ in the intestinal mucosa, and thickening rectum wall with necrosis and inflammation were scored as three points; complete necrosis of the rectum wall and ulcer with a diameter of greater than $1 \mathrm{~cm}$ were scored as four points.

\section{Tissue damage index scoring}

Detailed scoring rules on tissue damage index (TDI) [8]: After $24 \mathrm{~h}$ of successful modeling, the lesions of mice were fixed with $100 \mathrm{~mL} / \mathrm{L}$ formaldehyde and imbedded using paraffin to prepare paraffin sections. The sections were stained with hematoxylin and eosin (HE). The morphology of sections was observed under an optical microscope, and TDI was scored. The scoring standards of TDI mainly included inflammatory cell infiltration, the depth of infiltration, and the depth of an intestinal ulcer. No inflammatory cell infiltration of zero; mild inflammatory cell infiltration of one point; severe inflammatory cell infiltration of two points. The depth of infiltration was scored as follows: Infiltration to the mucosal layer of one point; infiltration to the mucosa and submucosa of two points; infiltration to the entire colon layer of three points. The depth of intestinal ulcer was scored as follows: No ulcer of zero; ulcer to the 
epithelium of one point; ulcer to the lamina propria mucosa of two points; ulcer to the muscularis mucosa of three points.

\section{ELISA}

The obtained peripheral blood was centrifuged at 3,000 rpm for $10 \mathrm{~min}$ at low temperature to collect the upper serum which was then centrifuged at $8,000 \mathrm{rpm}$ for $10 \mathrm{~min}$ at low temperature to collect the upper serum. The serum of mice was detected in strict accordance with the instruction of the ELISA kit (ebioscience, Thermo Fisher, USA). The ELISA kit was placed at room temperature for 20 min to prepare the washing solution. The blank well and sample well to be tested were set. After the standard substance was dissolved, $100 \mu \mathrm{L}$ washing solution were added to the sptting plate to make the standard curve. The sample of $50 \mu \mathrm{L}$ was added to the sample well to be tested, including $10 \mu \mathrm{L}$ samples to be tested and 40 $\mu \mathrm{L}$ diluent. After the coated plate was sealed, it was incubated at a constant temperature of $37^{\circ} \mathrm{C}$ for 30 min. The sample to be tested was washed after the plate was uncovered. The sample well, after patted dry, was added with $50 \mu \mathrm{L}$ biotinylated antibodies working solution and incubated at a constant temperature of $37^{\circ} \mathrm{C}$ for $30 \mathrm{~min}$. The sample to be tested was washed after the plate was uncovered. The sample well, after patted dry, was added with $50 \mu \mathrm{L}$ color-developing agent $A$ in a dark place, followed by the addition of $50 \mu \mathrm{L}$ color-developing agent $\mathrm{B}$. After the solution was mixed by gently shaking, the color was developed at $37^{\circ} \mathrm{C}$ for $15 \mathrm{~min}$. After finishing color development, $50 \mu \mathrm{L}$ stop buffers were immediately added. Optical density (OD) value at $450 \mathrm{~nm}$ in each well was measured using a microplate reader (BioTek Synergy 2, BioTek, USA). The contents $(\mathrm{pg} / \mathrm{mL}$ ) of tumor necrosis factor-a (TNF-a, ab6671, Abcam, USA), interleukin-4 (IL-4, ab11524, Abcam, USA), IL-8 (ab117318, Abcam, USA), IL-10 (ab108870, Abcam, USA), and TNF-Y (ab106099, Abcam, USA) in the serum of mice were analyzed through the standard curve drawn based on OD values.

\section{Cell cycle and apoptosis detection by flow cytometry}

The colon tissues of mice were put on a $60 \mu \mathrm{m}$-aperture nylon beaker, cut into pieces by an ophthalmological curved scissor, and washed with phosphate buffered saline (PBS). The single cells were collected, fixed with $95 \%$ ethanol, and washed with PBS once before centrifugation. Then centrifugation was performed at 1,500 rpm for $5 \mathrm{~min}$. The supernatant was discarded. Cell cycle detection: Cells were treated in dark place, and incubated in a water bath with the addition of $100 \mu \mathrm{L}$ RnaseA (SJ0709, Beijing Baiaolaibo Technology Co. Ltd., China) at $37^{\circ} \mathrm{C}$ for $30 \mathrm{~min}$ follow by the addition of $400 \mu \mathrm{L} \mathrm{PI} \mathrm{dye} \mathrm{solution} \mathrm{(Sigma,} \mathrm{USA).} \mathrm{After} \mathrm{cells} \mathrm{were} \mathrm{treated} \mathrm{in} \mathrm{dark} \mathrm{place} \mathrm{at} 4^{\circ} \mathrm{C}$ for $30 \mathrm{~min}$, the red fluorescence intensity at $488 \mathrm{~nm}$ in flow cytometer (CytoFLEX, Beckman Coulter, CA 92821, USA) was recorded to determine the cell cycle distribution in each group. Cell apoptosis detection: Annexin-V-FITC, $\mathrm{PI}$ and HEPES buffer at the ratio of 1:2:50 were prepared into Annexin-V-FITC/PI dye liquor according to the instruction of Annexin-V-FITC apoptosis assays kit (Sigma, USA). A total of $1 * 10^{6}$ cells were resuspended per $100 \mu \mathrm{L}$ dye liquor. The liquor was mixed by shaking and incubated at room temperature for $15 \mathrm{~min}$. Then the liquor was added with $1 \mathrm{~mL}$ HEPES buffer and mixed by shaking. The $525 \mathrm{~nm}$ and 
$620 \mathrm{~nm}$ band-pass filters were excited with the wavelength of $488 \mathrm{~nm}$ to detect FITC and PI fluorescence expressions. The apoptosis was measured through FITC and PI fluorescence expressions.

\section{qRT-PCR}

The colon tissues of mice were used to prepare tissue homogenate. Total RNA in the tissue homogenate was extracted using Trizol (10296028, Thermo fisher, USA), and the purity and concentration of sample total RNA were determined. Sample RNA was reversed to cDNA using the reverse transcription kit (11939823001, Merck, USA). qRT-PCR was performed with cDNA as the template. Total reaction solution was $10 \mu \mathrm{L}$, including $0.5 \mu \mathrm{L}$ PCR forward primer, $0.5 \mu \mathrm{L}$ PCR reverse primer, $1 \mu \mathrm{L}$ cDNA template, $3 \mu \mathrm{L}$ $\mathrm{ddH}_{2} \mathrm{O}$, and $5 \mu \mathrm{L}(2 \times)$ SYBR $^{\circledR}$ Premix Ex Taq ${ }^{\mathrm{TM}}$ II. qRT-PCR amplification procedure: pre-denaturation at $95^{\circ} \mathrm{C}$ for $4 \mathrm{~min}$, denaturation at $94^{\circ} \mathrm{C}$ for $30 \mathrm{~s}$ and annealing at $60^{\circ} \mathrm{C}$ for $30 \mathrm{~s}$ for 35 circles, setting the solubility curve, and extension at $72^{\circ} \mathrm{C}$ for $5 \mathrm{~min}$. The reference gene of qRT-PCR was GAPDH. The primers of Bax, Bcl-2, Caspase3, Fas, FasL and GAPDH were synthesized by the Sangon Biotech (Shanghai) Co., Ltd., China and shown in Table 1. Expressions of products were calculated using $2^{-\Delta \Delta C t}$. $\Delta \Delta C \mathrm{t}=\Delta \mathrm{Ct}_{\text {the rest groups }}-\Delta \mathrm{Ct}_{\text {control group }} . \Delta \mathrm{Ct}=\mathrm{Ct}_{\text {target gene }}-\mathrm{Ct}_{\mathrm{GAPDH}} \cdot \mathrm{Ct}$ represented the number of amplification cycles when the fluorescence intensity reached the threshold value, after qPCR was performed for samples to be tested.

Table 1 qRT-PCR primer sequence

\begin{tabular}{ll}
\hline Name & Sequence \\
\hline TrkB & Forward: 5'- CTGGGGCTTATGCCTGCTG-3' \\
& Reverse: 5'- AGGCTCAGTACACCAAATCCTA-3' \\
PLC & Forward: 5'- TTCCAGATGGTCTATTTCCGGG-3' \\
& Reverse: 5'- CTTGGCACTTGCATCCTCC-3' \\
IP3 & Forward: 5'- CGTTTTGAGTTTGAAGGCGTTT-3' \\
& Reverse: 5'- CATCTTGCGCCAATTCCCG-3' \\
Bax & Forward: 5'- AGACAGGGGCCTTTTTGCTAC-3' \\
& Reverse: 5'- AATTCGCCGGAGACACTCG-3' \\
Bcl-2 & Forward: 5'- GCTACCGTCGTGACTTCGC-3' \\
& Reverse: 5'- CCCCACCGAACTCAAAGAAGG-3' \\
Caspase3 & Forward: 5'- CTCGCTCTGGTACGGATGTG-3' \\
& Reverse: 5'- TCCCATAAATGACCCCTTCATCA-3' \\
\hline FasL & Forward: 5'- GCGGGTTCGTGAAACTGATAA-3' \\
& Reverse: 5'- GCAAAATGGGCCTCCTTGATA-3' \\
& Reverse: 5'- ATTTGTGTTGTGGTCCTTCTTCT-3' \\
& Forward: 5'- AGGTCGGTGTGAACGGATTTG-3' \\
& Forse: 5'- GGGGTCGTTGATGGCAACA-3' \\
\hline
\end{tabular}

TrkB, protein-tyrosine kinase receptor B; PLC, phospholipase C; IP3, inositol triphosphate. 


\section{Western blot}

The intestinal mucosa of mice was used to prepare tissue homogenate. The tissue was lysed on ice using RIPA to extract total protein. Centrifugation was performed at 13,000 rpm for 2 min to obtain the supernatant. After the protein concentration was adjusted using a BCA kit (P0012S, Beyotime Biotechnology Co., Ltd., Shanghai, China), $160 \mu \mathrm{L}$ supernatant were mixed with $40 \mu \mathrm{L} 5 \times$ SDS loading buffer. The mixture was put into a boiling water bath for $10 \mathrm{~min}$. Then SDS-PAGE was performed. The protein was transferred to PVDF membranes by wet method; the PVDF membrane was pretreated by immersing in methanol for $30 \mathrm{~s}$ and washing with $\mathrm{dd}_{2} \mathrm{O}$. The membrane was sealed with $5 \%$ skimmed milk powder and incubated with primary antibodies at room temperature for $2 \mathrm{~h}$. The primary antibodies included rabbit polyclonal antibodies p-TrkB (ab109684, 1/2,000, abcam, USA), TrkB (ab18987, 1/2,000, abcam, USA), PLC-ү1 (ab107455, 1/2,000, abcam, USA), PLC (ab154610, 1/3,000, abcam, USA), IP3 (ab5804, 1/1,000, abcam, USA), TNF-a (ab6671, 1/2,000, abcam, USA), TNF-y (ab19139, 1/1,000, abcam, USA), IL-4 (ab9728, 1/2,000, abcam, USA), IL-8 (ab7747, 1/1,000, abcam, USA), IL-10 (ab6696, 1/3,000, abcam, USA), Bax (ab32503, 1/5,000, abcam, USA), Bcl-2 (ab182858, 1/2,000, abcam, USA), Caspase3 (ab13847, 1/500, abcam, USA), Fas (ab82419, 1/1,000, abcam, USA), FasL (ab15285, 1/2,000, abcam, USA), and GAPDH (ab181602, 1/10,000, abcam, USA). Then the membrane was rinsed with tris buffered saline tween (TBST) three times, 5-10 min every time. The membrane was incubated with second antibody goat anti-rabbit IgG (ab6721, 1/2,000, abcam, USA) at room temperature for $2 \mathrm{~h}$. Then the membrane was rinsed with TBST three times, 5-10 min every time. The PVDF membrane was placed in an E-gel imager and covered using developing agent. The protein was photographed through Bio-Rad image analysis system (ChemiDoc MP, BIO-RAD, USA). The gray value of the protein band was analyzed by Quantity One software. The relative content of protein was calculated by the gray value of the protein band / the gray value of the internal reference band.

\section{Statistical analysis}

SPSS 21.0 software (SPSS, Inc, Chicago, IL, USA) was used to analyze the data. All measurement data were represented as mean \pm standard deviation. Comparison among groups was performed using oneway ANOVA and post hoc test, Bonferroni pairwise comparison. There was a significant difference at $P<0.05$.

\section{Results}

\section{DAl, CMDI and TDI scores}

DAl score of mice in normal, control, model, and K252a groups was shown in Fig. 1a. There was no significant difference in DAI score between normal group and control group ( $P>0.05)$. DAl score in model and K252a groups was significantly increased as compared to normal group (both $P<0.05$ ). DAl score in K252a group was significantly higher than that in model group $(P<0.05)$. 
CMDI score of mice in normal, control, model, and K252a groups was shown in Fig. 1b. There was no significant difference in CMDI score between normal group and control group ( $P>0.05)$. CMDI score in model and K252a groups was significantly increased as compared to normal group (both $P<0.05$ ). CMDI score in $\mathrm{K} 252 \mathrm{a}$ group was significantly higher than that in model group $(P<0.05)$.

TDI score of mice in normal, control, model, and K252a groups was shown in Fig. 1c. There was no significant difference in TDI score between normal group and control group ( $P>0.05)$. TDI score in model and K252a groups was significantly increased as compared to normal group (both $P<0.05$ ). TDI score in $\mathrm{K} 252 \mathrm{a}$ group was significantly higher than that in model group $(P<0.05)$.

\section{Contents of related inflammatory factors}

The contents of TNF-a, TNF-y, IL-4, IL-8 and IL-10 in the serum of mice in normal, control, model and K252a groups were detected by ELISA (Fig. 2). Compared with normal group, there were no significant differences in the content of TNF- $a$ and TNF- $y$ in the rest groups (both $P>0.05$ ), and there were a significant increase of IL-4 and IL-8 contents and a significant decrease of IL-10 content in model and K252a groups (all $P<0.05$ ). There were a significant increase of IL-4 and IL-8 contents and a significant decrease of IL-10 content in K252a group as compared to model group (all $P<0.05$ ). There were no significant differences between control group and normal group ( $P>0.05)$.

\section{Protein expressions of related inflammatory factors in the colon tissues}

Protein expressions of inflammatory factors in the colon tissues were measured by Western blot (Fig. 3). Compared with normal group, there were no significant differences in protein expressions of TNF- $a$ and TNF- $y$ in the rest groups (both $P>0.05$ ), and there were a significant increase of IL-4 and IL-8 protein expressions and a significant decrease of IL-10 protein expression in model and K252a groups (all $P<0.05)$. There were a significant increase of IL-4 and IL-8 protein expressions and a significant decrease of IL-10 protein expression in K252a group as compared to model group (all $P<0.05$ ). There were no significant differences between control group and normal group ( $P>0.05)$.

\section{Enterocyte cell cycle distributions}

Enterocyte cell cycle distribution in normal, control, model and K252a groups was detected by flow cytometry (Fig. 4). There were no significant differences in cell proportion in all phases between control group and normal group ( $P>0.05)$. Compared with normal group, there were a significant increase of cell proportion in the G0/G1 phase and a significant decrease of cell proportion in the $S$ phase in model and K252a groups (all $P<0.05$ ). There were a significant increase of cell proportion in the $\mathrm{G} 0 / \mathrm{G} 1$ phase and a significant decrease of cell proportion in the $\mathrm{S}$ phase in $\mathrm{K} 252 \mathrm{a}$ group as compared to model group (all $P<0.05)$. There were no significant differences in cell proportion in the $\mathrm{G} 2 / \mathrm{M}$ phase among the four groups $(P>0.05)$.

\section{Enterocyte apoptosis}


Enterocyte apoptosis in normal, control, model and K252a groups were detected by flow cytometry (Fig. $5)$. There was no significant difference in apoptotic rate between control group and normal group ( $P>0.05)$. Compared with normal group, there was a significant increase of apoptotic rate in model and $\mathrm{K} 252 \mathrm{a}$ groups $(P<0.05)$. There was a significant increase of apoptotic rate in $\mathrm{K} 252 \mathrm{a}$ group as compared to model group $(P<0.05)$.

\section{mRNA expression of apoptosis gene in TrkB-PLC/IP3 pathway in the colon tissue}

mRNA expressions of TrkB, PLC, IP3, Bax, Caspase3, Bcl-2, FasL and Fas in the colon tissue were measured by qRT-PCR (Fig. 6a). There was no significant difference between control group and normal group ( $P>0.05$ ). Compared with normal group, there was a significant increase of Bax, Caspase3, FasL and Fas mRNA expressions and a significant decrease of TrkB, PLC, IP3 and Bcl-2 mRNA expressions in model and K252a groups (all $P<0.05$ ). There was a significant increase of Bax, Caspase3, FasL and Fas mRNA expressions and a significant decrease of TrkB, PLC, IP3 and Bcl-2 mRNA expressions in K252a group as compared to model group (all $P<0.05$ ).

Protein expressions of p-TrkB, PLC-y1, IP3, Bax, Caspase3, Bcl-2, FasL and Fas in the colon tissue were measured by Western blot (Fig. 6b, c). There was no significant difference between control group and normal group ( $P>0.05)$. Compared with normal group, there was a significant increase of Bax, Caspase3, FasL and Fas protein expressions and a significant decrease of p-TrkB/TrkB, PLC- 1 1/PLC, IP3 and Bcl-2 protein expressions in model and $\mathrm{K} 252 \mathrm{a}$ groups (all $P<0.05$ ). There was a significant increase of $\mathrm{Bax}$, Caspase3, FasL and Fas protein expressions and a significant decrease of p-TrkB/TrkB, PLC-y1/PLC, IP3 and $\mathrm{Bcl}-2$ protein expressions in K252a group as compared to model group (all $P<0.05$ ).

\section{Discussion}

Colitis, as a kind of recurrent chronic colitis, is listed as a modern refractory disease due to obstinacy and easy recurrence $[20,21]$. Until now, there are many studies on the clinical drug development and treatment for colitis, while there are few studies on the related mechanisms. Therefore, it is of great significance to explore the mechanism of colitis.

TrkB as an acceptor molecule is rapidly activated by phosphorylation after receiving the upstream signal. By activating the downstream PLC/IP3 pathway, the activated TrkB promotes the increase of intracellular calcium ion concentration, thus facilitating the excitation of intestinal nerve cells and the conduction of excitation, and promoting intestinal motility $[22,23]$. At present, studies on TrkB-PLC/IP3 pathway mainly include antidepressant effect and intestinal motility promotion effect, and the improvement effect of TrkB-PLC/IP3 pathway on colitis has not been reported. In this study, sensitization and clysis were performed simultaneously on mice using oxazolone to establish colitis mice models [24]. DAI, TDI and $\mathrm{CMDI}$ score in $\mathrm{K} 252 \mathrm{a}$ group was the highest and these scores in model group were higher significantly than those in normal and control groups. TrkB-PLC/IP3 pathway inhibitor increased DAI, TDI and CMDI score of mice with colitis. The contents of TNF- $y$, IL-4, IL-8, TNF- $a$ and IL-10 in the serum of mice were detected by ELISA; protein expressions of TNF- $y$, IL-4, IL-8, TNF- $a$ and IL-10 in the colon tissues were 
determined by Western blot. The results showed that there were no significant differences in the contents and protein expressions of TNF- $y$ and TNF-a, and there were a significant increase of IL-4 and IL-8 contents and protein expressions and a significant decrease of IL-10 content and protein expression in model and K252a groups. It indicated that the TrkB-PLC/IP3 pathway might have no significant effect on Th1 cytokines of colitis mice, and it mainly affected the expressions of Th2 cytokines IL-4, IL-8 and IL-10 to break Th1/Th2 cytokine balance, facilitating the development of inflammation. At present, no studies have confirmed the regulatory mechanism of TrkB-PLC/IP3 pathway on the inflammatory development of colitis. However, because the upstream BDNF of TrkB-PLC/IP3 pathway can improve the content of TNF-a and other inflammatory factor in depression, we speculated that the TrkB-PLC/IP3 pathway, as the downstream effector of BDNF, could also inhibit the inflammatory development in colitis [25-28].

Enterocyte cell cycle distribution and apoptosis were detected by flow cytometry [29-32]. Mice in model group and K252a group suffered cell cycle arrest and significantly increased apoptotic rate. Moreover, cell cycle arrest and apoptotic rate were significantly greater in K252a group than in model group. mRNA expressions of Bcl-2, FasL, Fas, Caspase3 and Bax were measured by qRT-PCR; protein expressions of upstream and middle signal factors TrkB, Bcl-2, Caspase3, FasL, Fas and Bax in TrkB-PLC/IP3 pathway were detected by Western blot. mRNA and protein expressions of Bax, FasL, Fas and Caspase3 were significantly increased, and mRNA expressions of TrkB, PLC, IP3, Bcl-2 and protein expressions of p-TrkB, PLC- $y 1$, IP3 and Bcl-2 were significantly decreased in model and K252a groups. Moreover, both increased expressions and decreased expressions in K252a group were significantly more than those in model group. High enterocyte apoptotic rate which might be affected through Fas/FasL pathway further exacerbated the condition of colitis mice. Studies have shown that the TrkB-PLC/IP3 pathway can inhibit anxiety- and depression-like behaviors, mainly by inhibiting the apoptosis of hippocampal neurons [3335]. In this study, we did not explore the specific mechanism of TrkB-PLC/IP3 pathway on inflammation and apoptosis, and its application in clinical treatment remained to be further studied.

\section{Conclusions}

TrkB-PLC/IP3 pathway promotes the expressions of IL-10 by inhibiting IL-4 and IL-8 expressions to improve Th1/Th2 cytokine balance, reducing inflammation. And TrkB-PLC/IP3 pathway suppresses enterocyte apoptosis by inhibiting Fas/FasL pathway, improving symptoms of colitis.

\section{Abbreviations}

TrkB, tyrosine kinase receptor B; PLC, phospholipase C; IP3, inositol triphosphate; BDNF, neurotrophic factor; $\mathrm{DAl}$, disease activity index; $\mathrm{CMDI}$, colon mucosa damage index; TDI, tissue damage index. TNF, tumor necrosis factor; IL, interleukin; HE, hematoxylin and eosin; OD, optical density; PBS, phosphate buffered saline; TBST, tris buffered saline tween.

\section{Declarations}




\section{Ethics approval and consent to participate}

The protocol and procedures employed were ethically reviewed and approved by the Animal Ethics Committee of The Second Hospital of Jilin University (No.D20190013), and experiments were performed in accordance with relevant institutional and national guidelines for the care and use of laboratory animals.

\section{Consent for publication}

Not applicable.

\section{Availability of data and material}

All data generated or analysed during this study are included in this published article.

\section{Competing interests}

The authors declare that there are no conflicts of interest.

\section{Funding}

This work was supported by the Key Scientific and Technological Projects of Jilin Province Science and Technology Department [20170204059SF] and the 13th Five-Year Science and Technology Research Fund of Jilin Province Education Department [JJKH20180097KJ]. The funding body did not play a role in study design, analysis, interpretation of the data, or the writing of the manuscript.

\section{Authors' contributions}

DLH and XGM designed the study, HHQ, XQL and LYJ performed the experiments, XQL and LYJ acquired the data, SYJ and HHQ performed the statistical analysis, XGM and SYJ compiled the literature sources, XGM and SYJ were the major contributor in writing the manuscript. All authors read and approved the manuscript.

\section{Acknowledgements}

Not applicable.

\section{References}

1. Colombel JF, Sands BE, Rutgeerts P, Sandborn W, Danese S, D'Haens G, et al. The safety of vedolizumab for ulcerative colitis and Crohn\"s disease. Gut. 2017;66(5):839-51.

2. Freeman HJ, James D, Mahoney CJ. Spontaneous Peritonitis from Perforation of the Colon in Collagenous Colitis. Canadian Journal of Gastroenterology. 2016;15(4):265-7. 
3. Li JH, Yu JP, Yu HG, Xu XM, Yu LL, Liu J, et al. Melatonin reduces inflammatory injury through inhibiting NF-kappaB activation in rats with colitis. Mediators of Inflammation. 2017;2005(4):185.

4. Chao K, Zhang S, Qiu Y, Chen X, Zhang X, Cai C, et al. Correction to: Human umbilical cord-derived mesenchymal stem cells protect against experimental colitis via CD5+ B regulatory cells. Stem Cell Research \& Therapy. 2016;10(1):109.

5. Harbord M, Eliakim R, Bettenworth D, Karmiris K, Katsanos K, Kopylov U, et al. Third European Evidence-based Consensus on Diagnosis and Management of Ulcerative Colitis. Part 2: Current Management. Journal of Crohns \& Colitis Supplements. 2017;11(7):769-84.

6. Bossa F, Latiano A, Rossi L, Magnani M, Santo ED, Palmieri O, et al. Erythrocyte-mediated delivery of dexamethasone in patients with mild-to-moderate ulcerative colitis, refractory to mesalamine: a randomized, controlled study. Journal of Crohns \& Colitis Supplements. 2016;1(1):23.

7. Magro F, Gionchetti P, Eliakim R, Ardizzone S, Armuzzi A, Barreiro-de Acosta M, et al. Third European Evidence-based Consensus on Diagnosis and Management of Ulcerative Colitis. Part 1: Definitions, Diagnosis, Extra-intestinal Manifestations, Pregnancy, Cancer Surveillance, Surgery, and lleo-anal Pouch Disorders. Journal of Crohns \& Colitis Supplements. 2017;11(6):649-70.

8. Yu Y, Zhao T, Yang D. Cotransfer of regulatory T cells improve the therapeutic effectiveness of mesenchymal stem cells in treating a colitis mouse model. Experimental Animals. 2017;66(2):16776.

9. Rodríguez-Nogales A, Algieri F, Garrido-Mesa J, Vezza T, Utrilla MP, Chueca N, et al. Differential intestinal anti-inflammatory effects of Lactobacillus fermentum and Lactobacillus salivarius in DSS mouse colitis: impact on microRNAs expression and microbiota composition. Molecular Nutrition \& Food Research. 2017;61(11):1700144.

10. Wang F, Johnson RL, Snyder PW, DeSmet ML, Fleet JC. An Inducible, Large-Intestine-Specific Transgenic Mouse Model for Colitis and Colitis-Induced Colon Cancer Research. Digestive Diseases \& Sciences. 2016;61(4):1069-79.

11. Igaki K, Komoike Y, Nakamura Y, Watanabe T, Yamasaki M, Fleming P, et al. MLN3126, an antagonist of the chemokine receptor CCR9, ameliorates inflammation in a T cell mediated mouse colitis model. International immunopharmacology. 2018;60:160-9.

12. Wu J, Mcduffie JE, Song J, Harris MC, Sun S. LC/MS/MS Profiling of Tissue Oxysterols and its Application in Dextran Sodium Sulphate Induced Mouse Colitis Models. Current Topics in Medicinal Chemistry. 2017;17(24):2781.

13. Palamides $P$, Jodeleit $H$, Föhlinger $M$, Beigel F, Herbach $N$, Mueller T, et al. Characterization and validation of a mouse model for colitis ulcerosa based on NOD-scid IL2R ynull mice reconstituted with peripheral blood mononuclear cells from patients. Disease Models \& Mechanisms. 2016;9(9):dmm.025452.

14. Cazarin C, Nogales AR, Algieri F, Utrilla MP, Rodríguez-Cabezas ME, Garrido-Mesa J, et al. Intestinal anti-inflammatory effects of Passiflora edulis peel in the dextran sodium sulphate model of mouse colitis. Journal of Functional Foods. 2016;26(565-576). 
15. Bitzer ZT, Elias RJ, Vijay-Kumar M, Lambert JD. (-)-Epigallocatechin-3-gallate decreases colonic inflammation and permeability in a mouse model of colitis, but reduces macronutrient digestion and exacerbates weight loss. Molecular Nutrition \& Food Research.

16. Guo T, Lin Q, Li X, Nie Y, Wang L, Shi L, et al. Octacosanol attenuates inflammation in both RAW264.7 macrophages and a mouse model of colitis. Journal of Agricultural \& Food Chemistry. 2017;65(18):3647-58.

17. Zhang H, Chen W. Interleukin 6 inhibition by triptolide prevents inflammation in a mouse model of ulcerative colitis. Experimental \& Therapeutic Medicine. 2017;14(3).

18. Park JS, Choi JW, Jhun JY, Ji YK, Cho ML. Lactobacillus acidophilus Improves Intestinal Inflammation in an Acute Colitis Mouse Model by Regulation of Th17 and Treg Cell Balance and Fibrosis Development. Journal of Medicinal Food. 2018;21(3).

19. Shepherd C, Giacomin P, Navarro S, Miller C, Loukas A, Wangchuk P. A medicinal plant compound, capnoidine, prevents the onset of inflammation in a mouse model of colitis. Journal of Ethnopharmacology. 2018;211:17-28.

20. Pott J, Kabat AM, Maloy KJ. Intestinal Epithelial Cell Autophagy Is Required to Protect against TNFInduced Apoptosis during Chronic Colitis in Mice. Cell Host \& Microbe. 2018;23(2):S193131281730553X.

21. Vendramini-Costa DB, Alcaide A, Pelizzaro-Rocha KJ, Talero E, ávila-Román J, Garcia-Mauri?o S, et al. Goniothalamin prevents the development of chemically induced and spontaneous colitis in rodents and induces apoptosis in the HT-29 human colon tumor cell line. Toxicology \& Applied Pharmacology. 2016;300:1-12.

22. Lai KO, Ip NY. Methods to Study the Signal Transduction of the Surface Receptor Tyrosine Kinase TrkB in Neurons. 2018;1722:211.

23. Gaydukov AE, Akutin IA, Bogacheva PO, Balezina OP. Changes in the Parameters of Quantal Acetylcholine Release after Activation of PAR1-Type Thrombin Receptors at the Mouse Neuromuscular Junctions. 2018;12(1):33-42.

24. Fei L, Xu K. Zhikang Capsule ameliorates dextran sodium sulfate-induced colitis by inhibition of inflammation, apoptosis, oxidative stress and MyD88-dependent TLR4 signaling pathway. Journal of Ethnopharmacology. 2016;192:236-47.

25. Xia C, Shen S, Hashmi F, Qiao LY. Colitis-induced bladder afferent neuronal activation is regulated by BDNF through PLCY pathway. Psychopharmacology (Berl). 2016;285(Pt B):126-35.

26. Zalli A, Jovanova O, Hoogendijk WJ, Tiemeier H, LA C. Low-grade inflammation predicts persistence of depressive symptoms. Psychopharmacology (Berl). 2016;233(9):1669-78.

27. Xiang L, Ren Y, Li X, Zhao W, Song Y. MicroRNA-204 suppresses epileptiform discharges through regulating TrkB-ERK1/2-CREB signaling in cultured hippocampal neurons. Brain Research. 2016;1639:99-107.

28. Chen $X$, Wang $X$, Tang L, Wang J, Shen C, Liu J, et al. Nhe5 deficiency enhances learning and memory via upregulating Bdnf/TrkB signaling in mice. Am J Med Genet B Neuropsychiatr Genet. 
2017;174(8):828-38.

29. Miao, Xianjing, Zhang, Dongmei, Zhao, Weijuan, et al. Sam68 modulates apoptosis of intestinal epithelial cells via mediating NF-kappa B activation in ulcerative colitis. Molecular Immunology. 2016;75:48-59.

30. Wu F, Huang Y, Dong F, Kwon JH. Ulcerative Colitis-Associated Long Noncoding RNA, BC012900, Regulates Intestinal Epithelial Cell Apoptosis. Inflammatory Bowel Diseases. 2016;22(4):782.

31. Han J, Zhao Q, Basmadjian C, Désaubry L, Theiss AL. Flavaglines Ameliorate Experimental Colitis and Protect Against Intestinal Epithelial Cell Apoptosis and Mitochondrial Dysfunction. Inflammatory Bowel Diseases. 2015;22(1).

32. Cui X, Shan X, Qian J, Ji Q, Wang L, Wang X, et al. The suppressor of cytokine signaling SOCS1 promotes apoptosis of intestinal epithelial cells via p53 signaling in Crohn\"s Disease. Experimental \& Molecular Pathology. 2016;101(1):1-11.

33. Zhao DY, Zhang WX, Qi QQ, Long X, Li X, Yu YB, et al. Brain-derived neurotrophic factor modulates intestinal barrier by inhibiting intestinal epithelial cells apoptosis in mice. Physiological Research. 2018;67(3):475-85.

34. Pandey R, Rai V, Mishra J, Mandrah K, Roy SK, Bandyopadhyay S. From the Cover: Arsenic Induces Hippocampal Neuronal Apoptosis and Cognitive Impairments via an Up-Regulated BMP2/SmadDependent Reduced BDNF/TrkB Signaling in Rats. Toxicological Sciences. 2017;159(1):137-58.

35. Zong C, Nie X, Zhang D, Ji Q, Qin Y, Wang L, et al. Up regulation of glyoxylate reductase/hydroxypyruvate reductase (GRHPR) is associated with intestinal epithelial cells apoptosis in TNBS-induced experimental colitis. Pathology - Research and Practice. 2016;212(5):365-71.

\section{Figures}

a

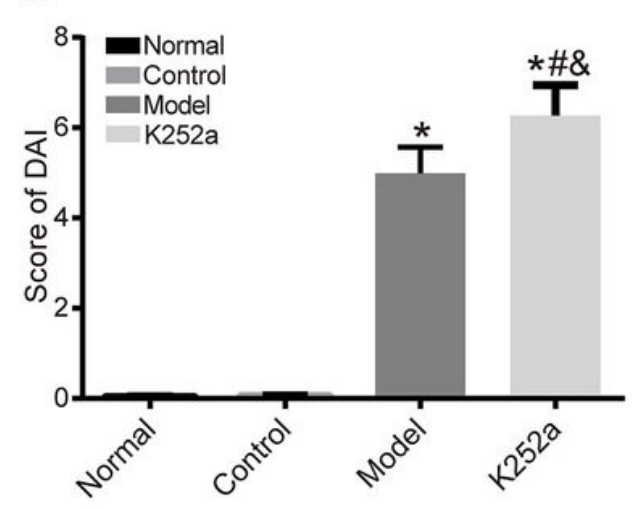

b

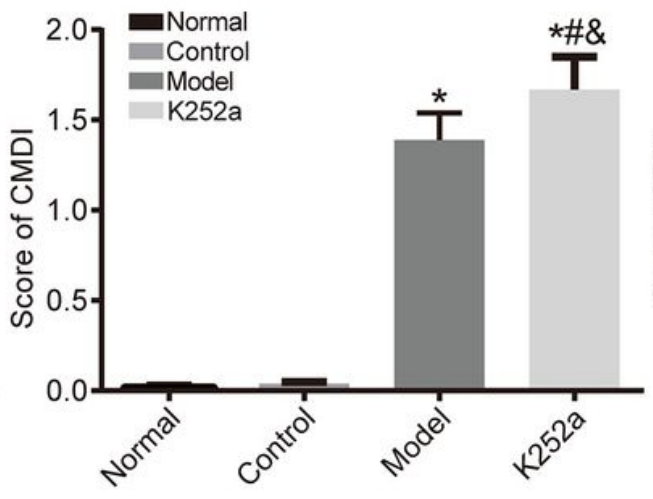

C

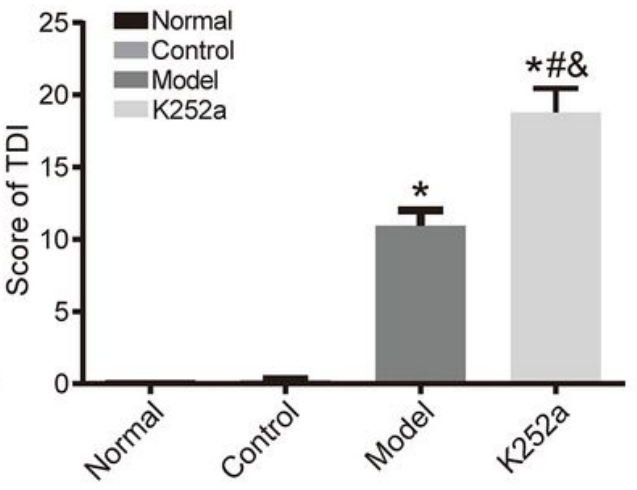

Figure 1

Scores comparison. (a) DAI score comparison. (b) CMDI score comparison. (c) TDI score comparison. Compared with normal group, ${ }^{*}<<0.05$; compared with control group, $\# \mathrm{P}<0.05$; compared with model 
group, \&P<0.05. DAl, disease activity index; CMDI, colon mucosa damage index; TDI, tissue damage index.

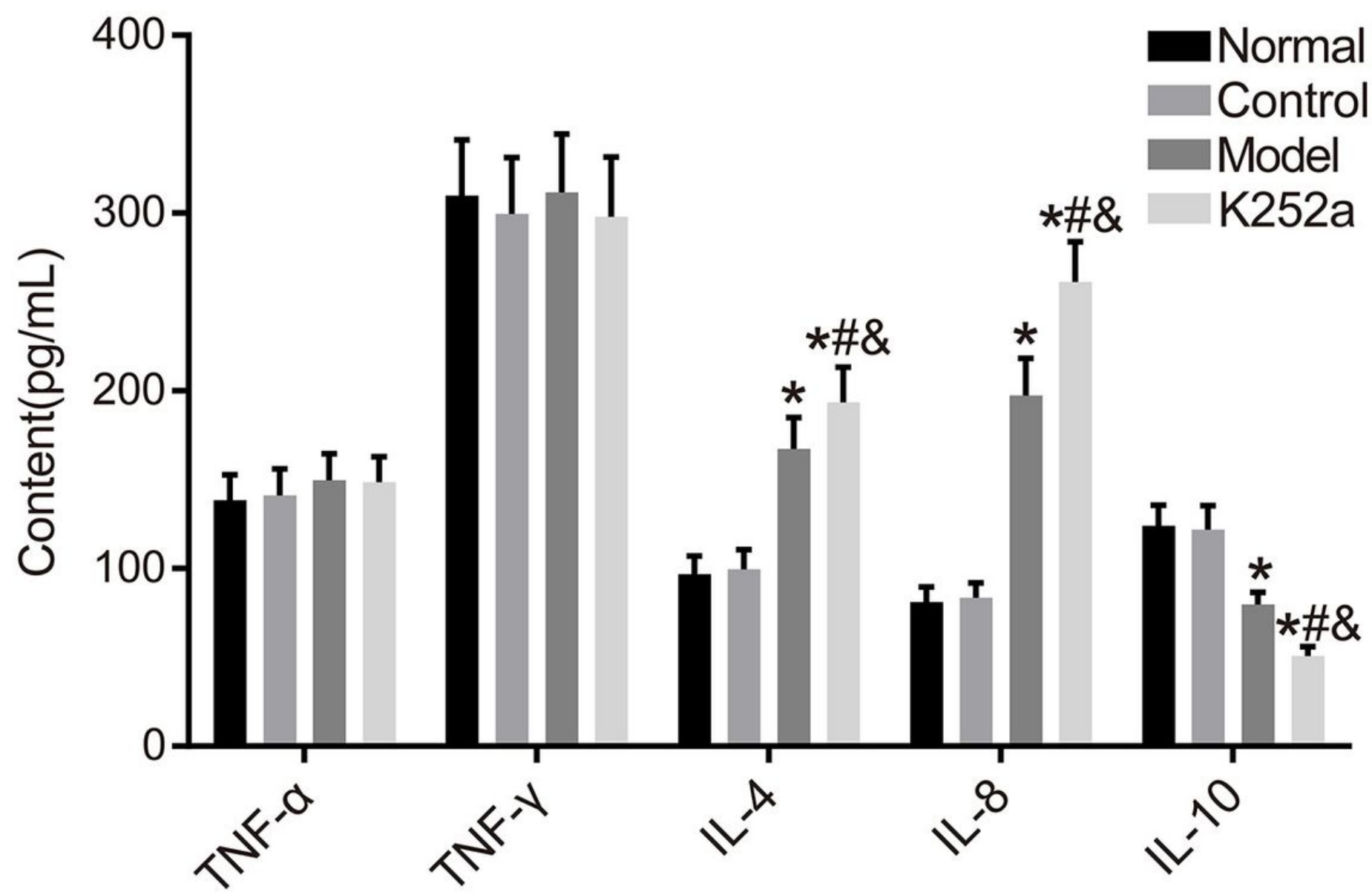

Figure 2

Contents of related inflammatory factors in the serum detected by ELISA. Compared with normal group, *P<0.05; compared with control group, \#P<0.05; compared with model group, \&P<0.05. TNF, tumor necrosis factor; IL, interleukin. 
a

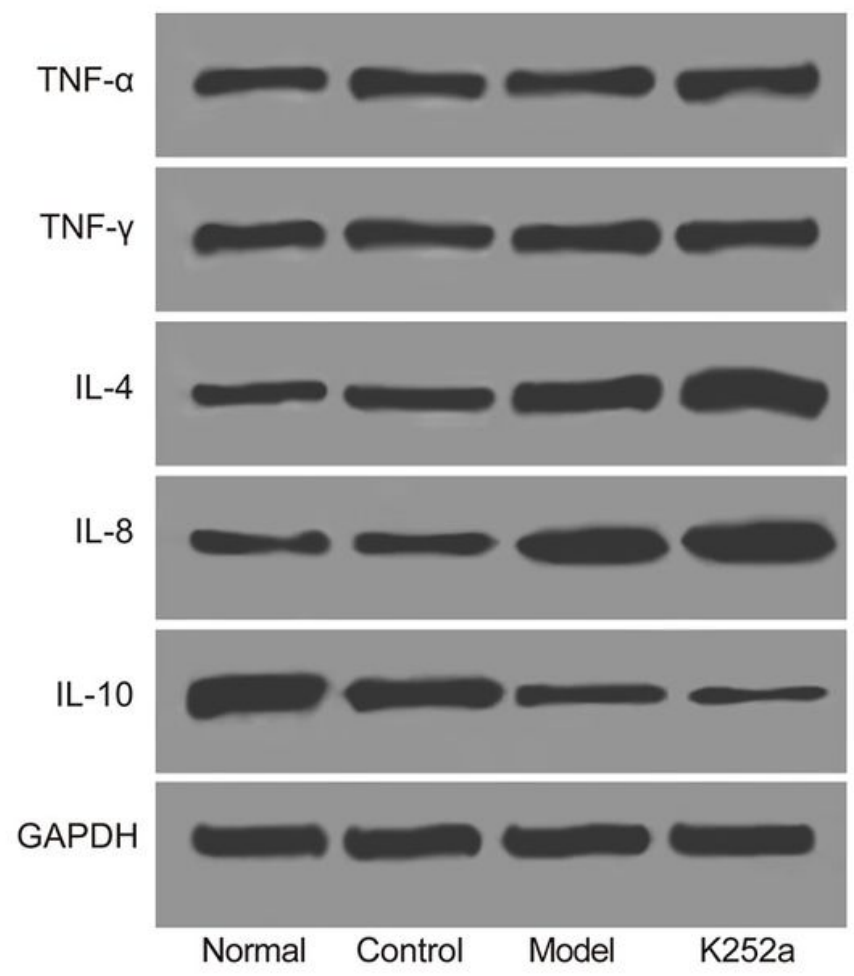

b

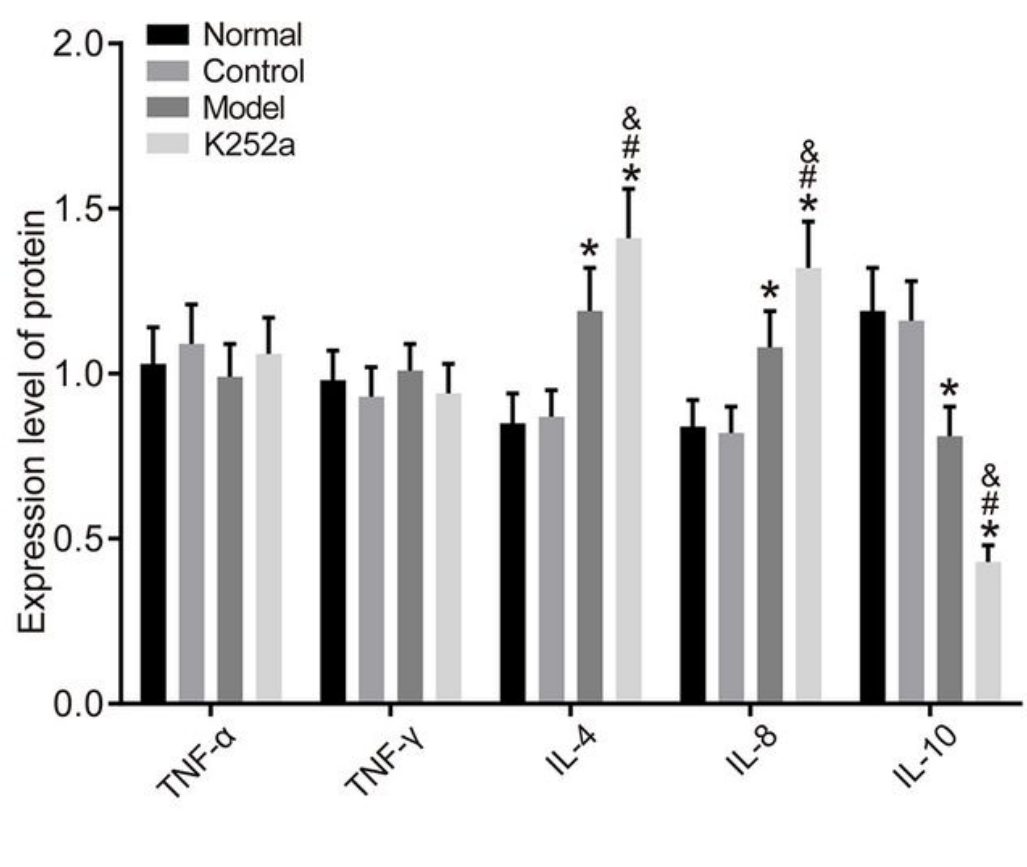

Figure 3

Protein expressions of inflammation-related genes in the colon tissues detected by western blot. (a) Protein bands. (b) Histogram of protein expression. Compared with normal group, ${ }^{*} \mathrm{P}<0.05$; compared with control group, $\# \mathrm{P}<0.05$; compared with model group, \&P<0.05. TNF, tumor necrosis factor; IL, interleukin. 
a
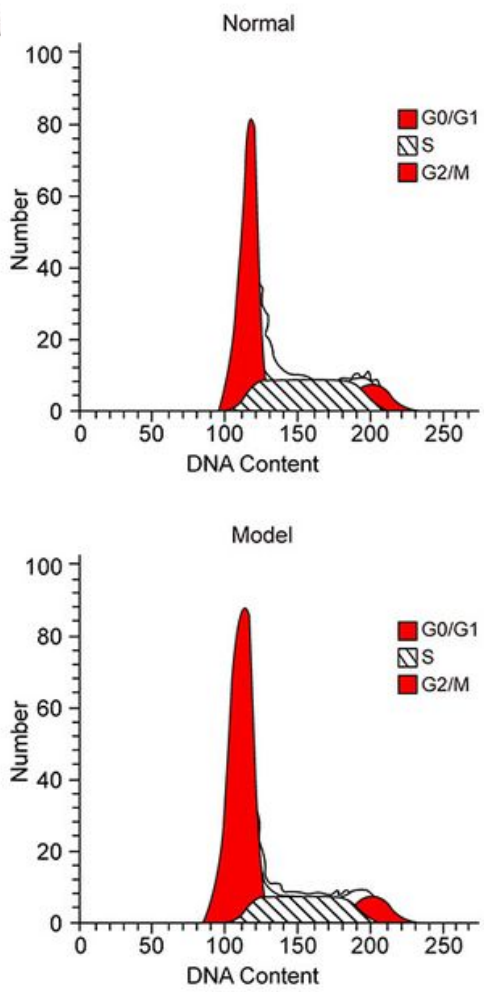
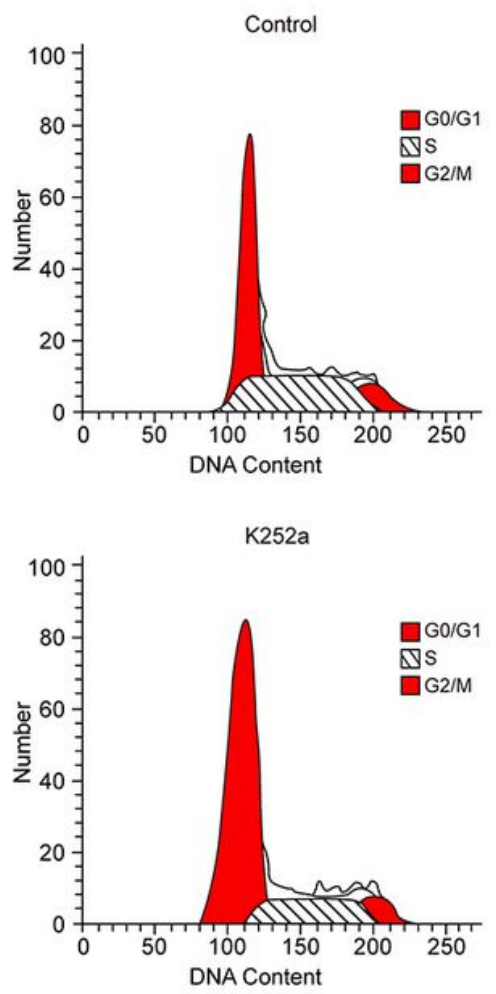

b

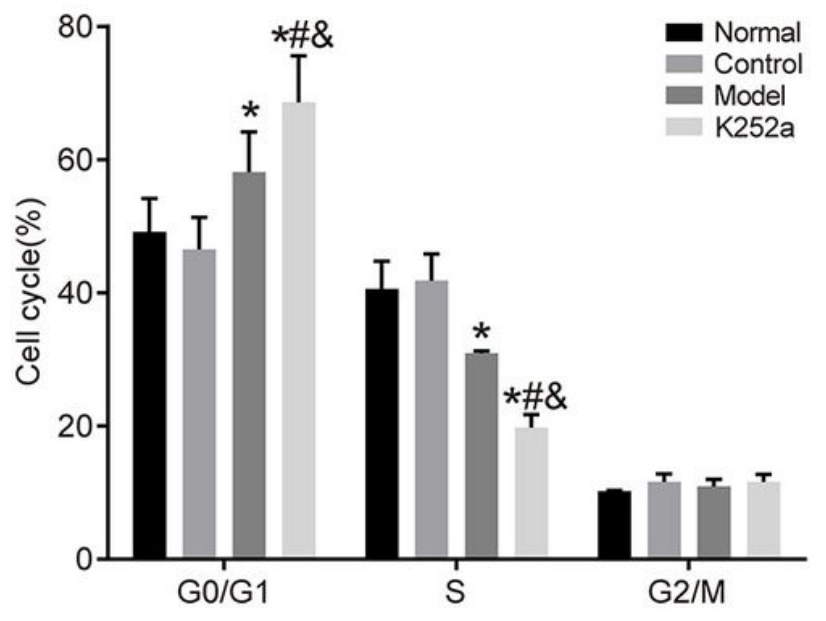

Figure 4

Enterocyte cell cycle distributions detected by flow cytometry. (a) Cell circle. (b) Cell circle distribution. Compared with normal group, ${ }^{*}<<0.05$; compared with control group, $\# \mathrm{P}<0.05$; compared with model group, \&P<0.05.

a
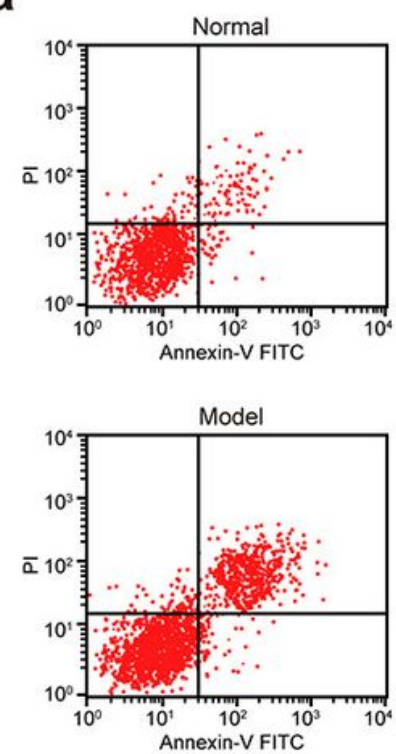
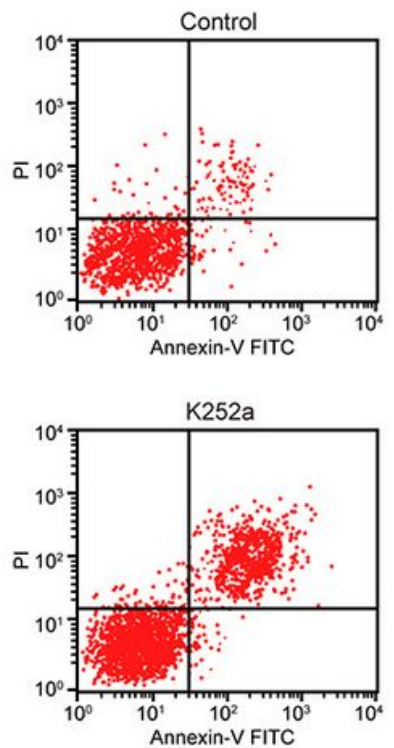

b

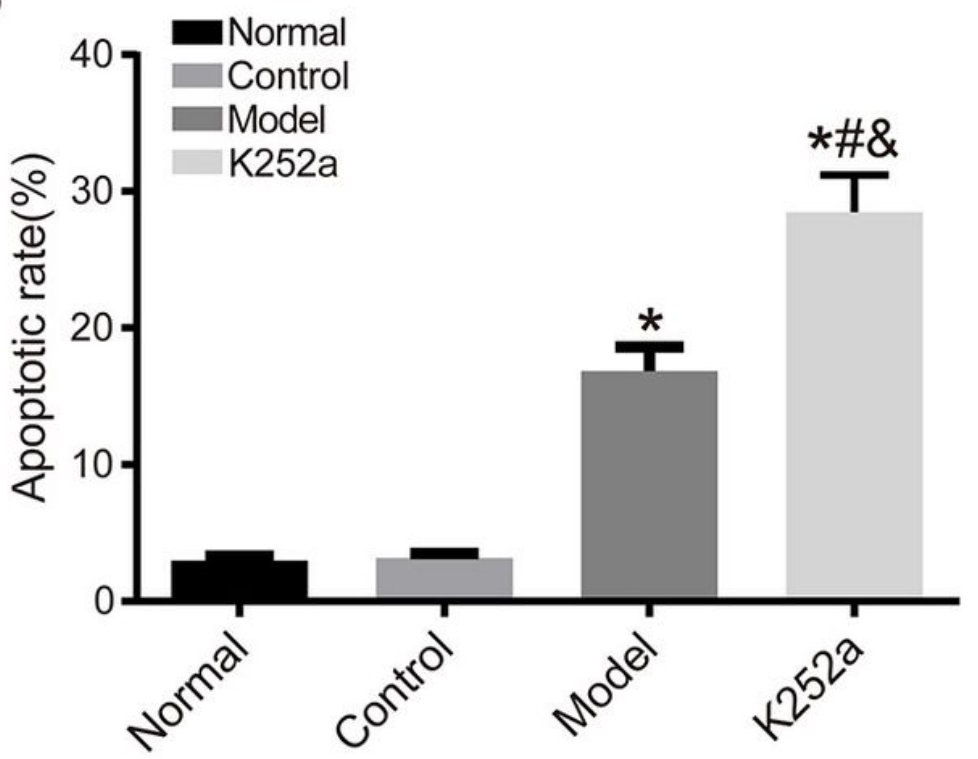

\section{Figure 5}


Enterocyte apoptosis detected by flow cytometry. (a) Apoptosis. (b) Histogram of enterocyte apoptosis rate. Compared with normal group, ${ }^{*} \mathrm{P}<0.05$; compared with control group, $\# \mathrm{P}<0.05$; compared with model group, \&P<0.05.
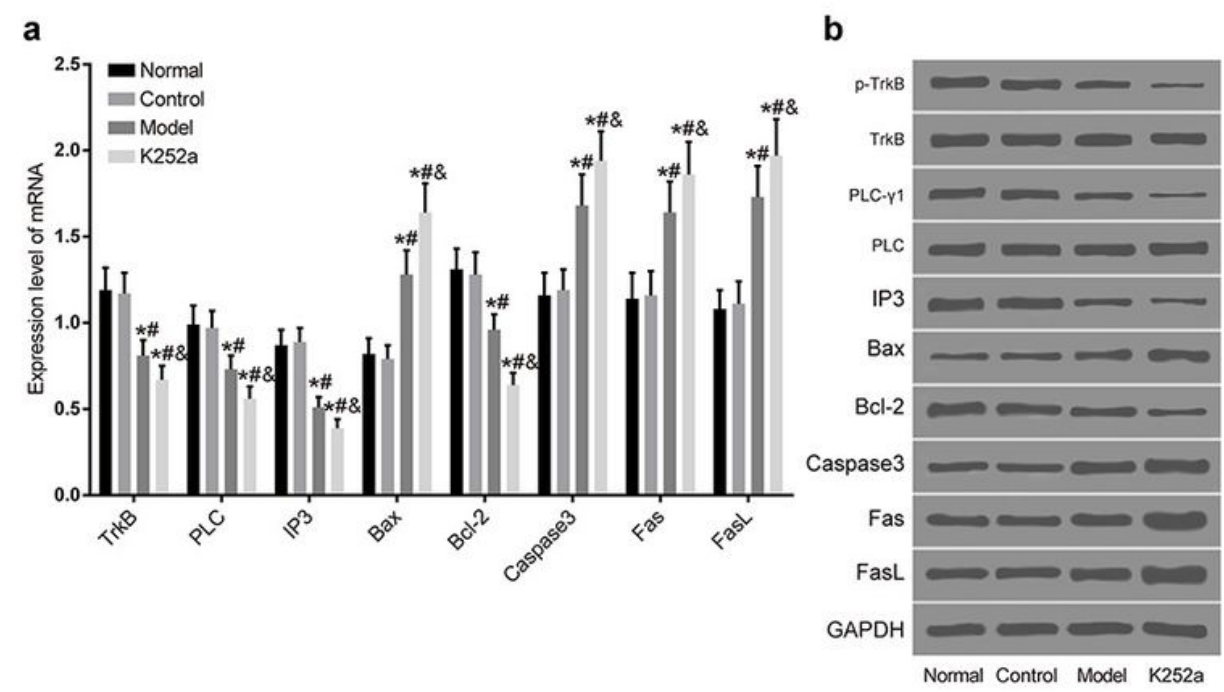

C

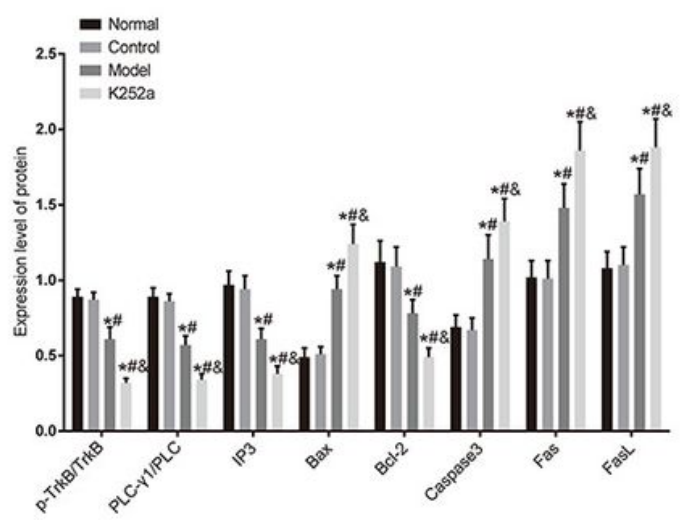

\section{Figure 6}

mRNA and protein expressions of related genes in the colon tissue detected by qRT-PCR and Western blot. (a) mRNA expressions of related genes in the colon tissue. (b) Protein bands. (c) Histogram of protein expressions. Compared with normal group, ${ }^{\star} \mathrm{P}<0.05$; compared with control group, $\# \mathrm{P}<0.05$; compared with model group, \&P<0.05. TrkB, tyrosine kinase receptor B; PLC, phospholipase C; IP3, inositol triphosphate.

\section{Supplementary Files}

This is a list of supplementary files associated with this preprint. Click to download.

- NC3RsARRIVEGuidelinesChecklistfillable.pdf 